Trauma Berufskrankh $2015 \cdot 17$ [Suppl 1]:83-88 DOI 10.1007/s10039-013-1988-8

Online publiziert: 14. September 2013

(c) Springer-Verlag Berlin Heidelberg 2013

\section{Kertai · B. Reingruber}

Klinik für Kinderchirurgie, Klinik St. Hedwig, Krankenhaus Barmherzige Brüder Regensburg

\section{Frakturen des} Unterarms beim Kind

\section{Möglichkeiten und Grenzen unterschiedlicher Therapiemethoden}

Der Unterarm stellt die häufigste Frakturlokalisation am wachsenden Skelett dar. Diese Verletzungen können meist leicht erkannt werden. Ebenso ist ihre Versorgung technisch häufig einfach und wird flächendeckend in Kliniken aller Versorgungsstufen durchgeführt. Die große Problematik dieser Frakturregion stellt die weit verbreitete Übertherapie dar. Die genaue Kenntnis und das Vertrauen auf die Möglichkeiten der spontanen Achskorrekturen im Wachstum sind essenziell, um den betroffenen Kindern unnötige Eingriffe zu ersparen. Im Folgenden werden die vermeintlich einfachen Frakturen des Unterarmschafts und des distalen Unterarms erläutert.

\section{Prävalenz}

Frakturen am Unterarm stellen mehr als die Hälfte aller Frakturen am wachsenden Skelett dar. Von diesen finden sich lediglich etwa 5\% (3-7\%) im Bereich des problematischen proximalen Unterarms. Etwa $20 \%(15-20 \%)$ betreffen den Unterarmschaft und etwa $2 / 3$ (50-80\%) den distalen Unterarm [3].

Bereits die Häufigkeit der Frakturen lässt vermuten, dass der ihnen zugrunde liegende Unfallmechanismus im Alltag häufig sein muss. Bei der Analyse der Ursachen von Unterarmfrakturen bewahrheitet sich dies: Mehr als die Hälfte davon ereignen sich beim Sport und Spielen, während Z. B. Verkehrsunfälle bezüglich der Ätiologie einen nur geringen Stellenwert haben [2].

\section{Frakturen des distalen Unterarms}

Sie stellen im Kindesalter die häufigste Frakturlokalisation überhaupt dar. Im Wesentlichen können 3 Frakturtypen unterschieden werden (• Tab. 1):

- die metaphysäre Wulst- oder Stauchungsfraktur,

- die rein metaphysäre Fraktur und

- Frakturen mit Beteiligung der Wachstumsfuge.

Darüber hinaus gibt es noch epiphysäre Frakturen mit Gelenkbeteiligung. Diese stellen jedoch eine absolute Rarität dar und bedürfen einer Versorgung wie beim Erwachsenen (unter Berücksichtigung der Wachstumsfuge), weshalb auf sie im
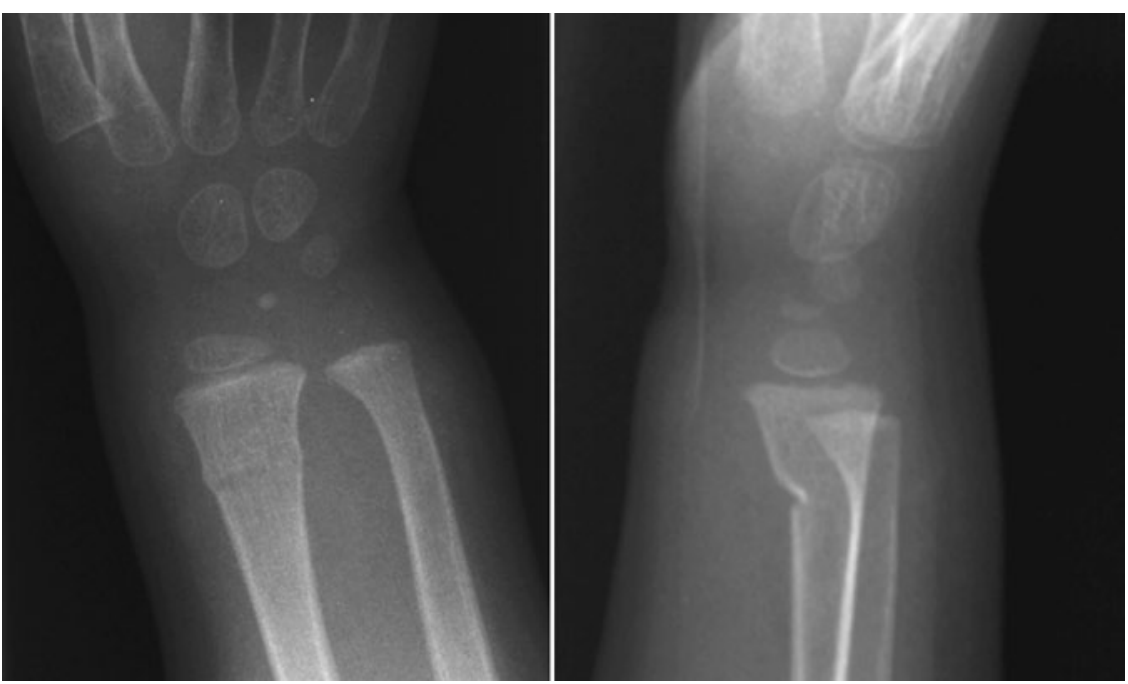

Abb. $1 \Delta$ Distale Radiuswulstfraktur mit dorsaler Einstauchung
Rahmen des vorliegenden Beitrags nicht weiter eingegangen wird.

\section{Metaphysäre Wulstfraktur}

Bei ihr kommt es durch eine axiale Krafteinwirkung zu einer Stauchung der Metaphyse ohne Unterbrechung der Kortikalis. Bei diesen Verletzungen ist keine wesentliche Achsabweichung vorhanden, und sie sind stets stabil (• Abb. 1, [12]). Deshalb benötigen sie nach der Diagnose mittels Röntgenaufnahmen in 2 Ebenen lediglich eine Ruhigstellung in einer Unterarmgipsschiene für 4 Wochen bei Kindern über 10 Jahren oder einer Oberarmgipsschiene für 3 Wochen bei jüngeren Kindern. Die Konsolidierungskontrolle erfolgt durch 


\section{Kindertraumatologie}


Abb. $2<$ Distale Unterarmfrakturen (von links nach rechts): komplette Fraktur von Ulna und Radius; komplette Fraktur des Radius und Fraktur des Processus styloideus ulnae; isolierte distale Radiusgrünholzfraktur
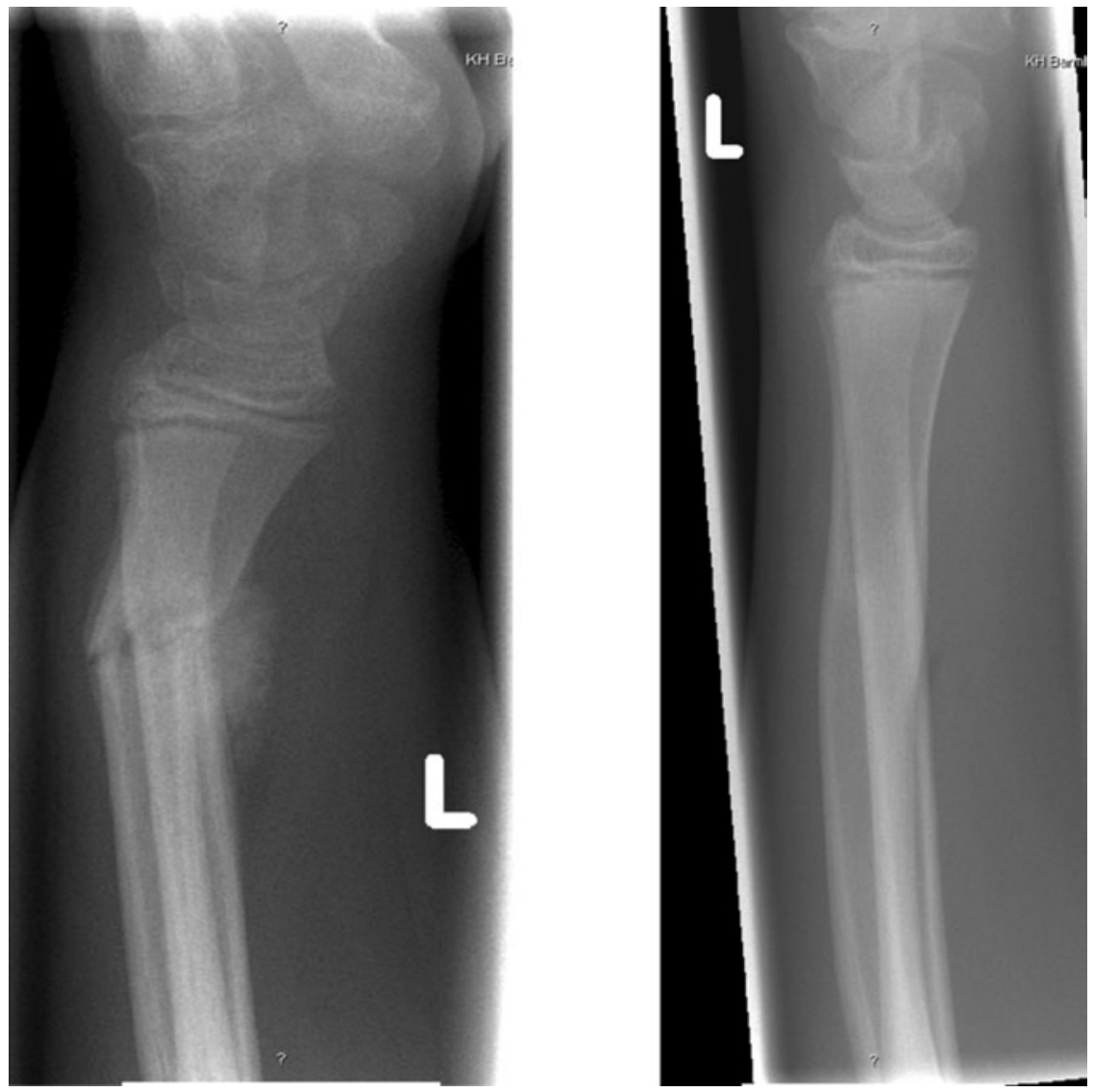

Abb. $3 \Delta 13$ Jahre alter Junge mit in $23^{\circ}$ Rekurvationsfehlstellung verheilter distaler metaphysärer Unterarmfraktur (links), fast vollständiges Remodelling nach nur 1 Jahr (rechts) das Ertasten des nicht druckdolenten Kallus ohne weitere Bildgebung.

\section{Distale metaphysäre Unterarmfrakturen}

Frakturen der distalen Unterarmmetaphysen können eine große Bandbreite unterschiedlicher Frakturtypen und -kombinationen aufweisen. So können beide Knochen oder aber nur der Radius frakturiert sein. Ebenso können ein Knochen komplett, der andere inkomplett oder beide in gleicher Weise betroffen sein ( $\bullet$ Abb. 2). Diese Differenzierungen spielen jedoch nur bezüglich der Beurteilung der Stabilität, nicht aber zur Feststellung einer Repositionsbedürftigkeit eine Rolle.

\section{Korrekturpotenzial}

Im Allgemeinen muss jedem Behandler stets bewusst sein, dass die distalen Unterarmmetaphysen Regionen enormer Korrekturfähigkeit darstellen und dieses Potenzial selbst von erfahrenen Kindertraumatologen häufig unterschätzt wird. Ursache für diese hohe Remodellierungspotenz sind der späte Fugenschluss $[4,6$, 8], der hohe Wachstumsanteil der distalen Fuge $(80 \%$ des Ulna- bzw. Radiuswachs- 
Tab. 1 Häufigkeitsverteilung der unter-

schiedlichen distalen Unterarmfrakturen

bei Kindern

Frakturtyp Häufigkeit (\%)

Wulst- oder Stauchungs- $\quad 40$

fraktur

Metaphysäre Frakturen $\quad 20$

Frakturen mit Fugen- $\quad 30$

beteiligung

Mischtypen 10

tums) und die Bewegung des Handgelenks in allen Ebenen.

Merke. Ab einem Alter von 10 Jahren wird die Grenzlinie bezüglich der Korrekturpotenz gezogen.

$\mathrm{Da}$ ein wesentlicher Bestandteil der Korrekturpotenz kindlicher Frakturen vom künftigen Wachstum abhängt, ist diese bei sehr jungen Kindern am höchsten und bei Adoleszenten am geringsten. Da es aber nicht möglich ist, leicht verständliche Empfehlungen für jedes Alter und jeden körperlichen Entwicklungsstand $\mathrm{zu}$ geben, wird üblicherweise ein Alter von 10 Jahren als Grenze definiert. Bei älteren Kindern stellen Achsabweichungen über $10^{\circ}$ eine Operationsindikation dar. Der erfahrene Kindertraumatologe wird beispielsweise auch einen 11-jährigen präpubertären Jungen eher großzügiger konservativ behandeln.

Bei Kindern unter 10 Jahren können Achsabweichungen bis $40^{\circ}$ in Ante- und Rekurvation sowie $20^{\circ}$ in Ulnar- und Radialdeviation belassen werden. Dislokationen bis zu einer gesamten Schaftbreite werden ebenso korrigiert wie selbst Verkürzungen eines der beiden paarigen Knochen [12]. Letzteres stellt jedoch aufgrund der deutlichen Instabilität meist dennoch eine Indikation zur Reposition dar. Bei den genannten Grenzwerten handelt es sich nicht um Grenzwerte der Korrekturfähigkeit, sondern sie stellen den Rahmen eines sicheren therapeutischen Bereichs dar. Wahrscheinlich könnten noch deutlich stärkere Achsabweichungen bis in ein höheres Alter toleriert werden ( $\bullet$ Abb. 3).

Für Kinder und Jugendliche über 10 Jahre werden $10^{\circ}$ Achsabweichung in allen Ebenen und eine Seitverschiebung um bis zu 1/3 der Schaftbreite als tolerabel angesehen.

Trauma Berufskrankh 2015 - 17[Suppl 1]:83-88 DOI 10.1007/s10039-013-1988-8

(c) Springer-Verlag Berlin Heidelberg 2013

\section{Kertai · B. Reingruber}

\section{Frakturen des Unterarms beim Kind. Möglichkeiten und Grenzen unterschiedlicher Therapiemethoden}

\section{Zusammenfassung}

Hintergrund. Frakturen des Unterarms sind die häufigsten Knochenbrüche im Kindesalter. Prinzipiell kann bezüglich der Lokalisation zwischen Frakturen des proximalen und des distalen Unterarms sowie des Unterarmschafts unterschieden werden. Letztere die Frakturen des distalen Unterarms sowie des Unterarmschafts - stellen über $90 \%$ aller Unterarmfrakturen dar und werden im vorliegenden Beitrag abgehandelt.

Therapie. Während es bei der Heilung von Knochenbrüchen des Unterarmschafts nur selten zu Achskorrekturen kommt, stellen die distale Radius- und Ulnametaphyse eine Region enormer Korrekturpotenz dar. Schaftfrakturen bedürfen daher meist einer Reposi- tion und Osteosynthese mit intramedullärem Nagel. Frakturen des distalen Unterarms dagegen können sehr häufig konservativ versorgt werden. Die Möglichkeiten der konservativen Behandlung werden hier oft unterschätzt, und selbst bei den wenigen Indikationen zur Operation wird häufig ein zu invasives Osteosyntheseverfahren gewählt. Ziel des vorliegenden Beitrags ist, den Leser in der bewusst zurückhaltenden Versorgung kindlicher Unterarmfrakturen zu bestärken.

\section{Schlüsselwörter}

Kind · Knochenbrüche · Radius · Ulna .

Osteosynthese

\section{Fractures of the forearm in children. Options and limits of various treatment methods}

\section{Abstract}

Background. Fractures of the forearm are the most common fractures in children. In general they can be distinguished by location between those of the proximal and distal forearm and those of the forearm diaphysis. The last two represent more than $90 \%$ of all forearm fractures and will be discussed in this article.

Therapy. While in the healing of fractures of the forearm shaft little correction of axis deviation is possible, the distal radius and ulna metaphyses represent regions of enormous correction potential. Shaft fractures therefore usually require reduction and internal fixa- tion with an intramedullary nail. Fractures of the distal forearm can very often be treated conservatively. The possibilities of conservative treatment are often underestimated and even with the few indications for surgery the method of fixation selected is often too invasive. This text aims to encourage the reader to choose a restrained therapy of pediatric forearm fractures.

\section{Keywords}

Child · Bone fracture $\cdot$ Radius · Ulna .

Osteosynthesis

\section{Frakturen mit Verletzungen der distalen Wachstumsfuge}

Epiphysenfugenverletzungen kommen bei Kindern insbesondere im Rahmen des pubertären Wachstumsschubs und der damit verbundenen Auflockerung der Fuge vor. Sie werden wie alle anderen Fugenverletzungen nach Aitken oder Salter u. Harris eingeteilt, wobei die Frakturform einer Epiphysenlösung mit metaphysärem Keil (Aitken I bzw. Salter-Harris II) bei weitem die häufigste ist.

Prinzipiell haben diese Frakturen ein ebenso hohes Korrekturpotenzial wie die zuvor beschriebenen metaphysären Frakturen, Seitverschiebungen bzw. Fugenlösungen sollten jedoch maximal bis $\mathrm{zu} 1 / 3$ der Fugenbreite toleriert werden [13]. Da diese Frakturen häufig im fortgeschrittenen Alter auftreten, gelten jedoch meist die strengeren Toleranzgrenzen wie sie für Kinder über 10 Jahren bei den metaphysären Frakturen genannt wurden.

\section{Therapie}

\section{Kirschner-Draht-Osteosynthese}

Sollte sich bei der primären Diagnostik sowohl einer metaphysären Fraktur als 


\section{Kindertraumatologie}

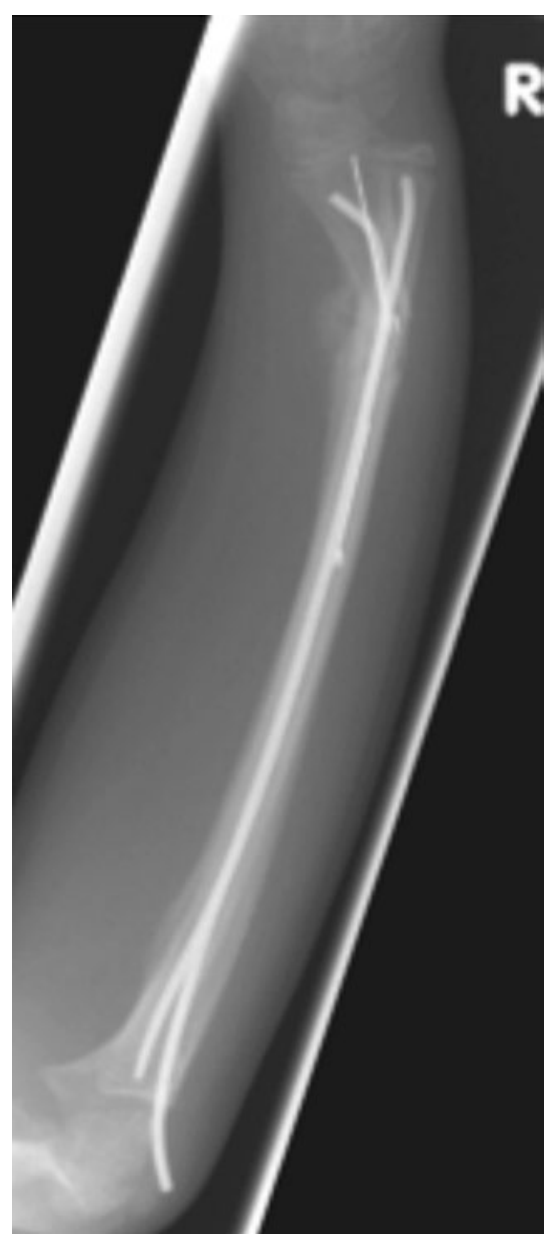

Abb. $4 \Delta$ Sekundäre Dislokation bei mit elastisch-stabiler intramedullärer Nagelung versorgter diametaphysärer Fraktur

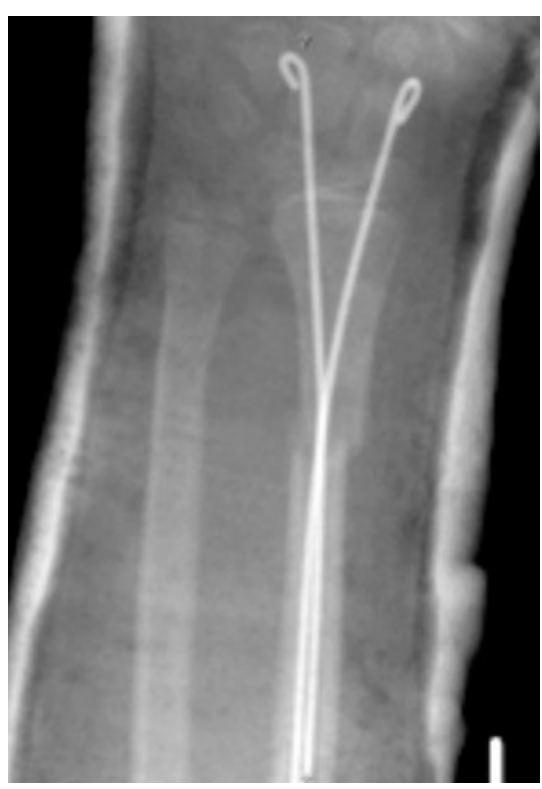

Abb. $5 \Delta$ Durch transepiphysäre, intramedulläre Drähte und Unterarmgips versorgte diametaphysäre Fraktur auch einer Fugenverletzung eine Indikation zur Reposition (nach oben genannten Kriterien) ergeben, empfehlen wir bei allen instabilen, also vollständigen, Frakturen eine Osteosynthese durchzuführen. Diese gelingt mit dem Kirschner-Draht stets, sodass ein anderes Osteosyntheseverfahren aufgrund der höheren Invasivität nicht gerechtfertigt ist. Die Reposition der Fraktur selbst kann fast ausnahmslos geschlossen erfolgreich durchgeführt werden.

In der Regel ist ein Draht, der über den Processus styloidus radii eingebracht wird, ausreichend. Gelegentlich ist ein weiterer, von dorsal eingebrachter Draht zum Erreichen der Stabilität notwendig. Obwohl die Kirschner-Draht-Osteosynthese ein relativ einfaches operatives Verfahren darstellt, ist insbesondere darauf zu achten, nicht durch die Randbereiche der Wachstumsfuge zu bohren, da in diesem Bereich die Ranvier-Zone [10] lokalisiert ist, in der die Differenzierung von Mesenchymzellen zu Chondroblasten stattfindet.

Lässt man die Drähte perkutan überstehen, ist keine weitere Narkose zur Metallentfernung notwendig. Postoperativ muss das Osteosyntheseergebnis mit einer Gipsschiene gesichert werden. Hierzu verwenden wir bis zum Alter von 10 Jahren einen Oberarm-, darüber hinaus einen Unterarmgips.

\section{Reine Reposition ohne Osteosynthese}

Alternativ zur Kirschner-Draht-Osteosynthese ist auch eine reine Reposition ohne Osteosynthese, wie wir sie bei dislozierten, jedoch stabilen Frakturen anwenden, auch bei instabilen Frakturen möglich. Bei der Wahl dieses Verfahrens ist jedoch explizit über das höhere Risiko einer sekundären Dislokation mit der Notwendigkeit einer Gipskeilung oder Rereposition aufzuklären.

Unserer Meinung nach sollte einer Frakturreposition in Narkose beim Kind eine definitive Versorgung folgen, sodass wir die Osteosynthese der instabilen Frakturen bevorzugen.

\section{Unterarmschaftfrakturen}

Hierbei können im Wesentlichen 2 Frakturtypen unterschieden werden:
- die komplette Unterarmfraktur und

- die Unterarmgrünholzfraktur.

Eine besondere Form der Unterarmfraktur stellt die Bowing-Fraktur, also der Biegungsbruch, dar, bei dem die Kortikalis an keiner Stelle unterbrochen, der Knochen jedoch in sich verbogen ist.

Häufig wird am Unterarmschaft eine Mischform festgestellt, bei der z. B. einer der paarigen Knochen intakt und der andere frakturiert ist.

\section{Begrenzte Spontankorrekturmöglichkeiten}

Im Gegensatz zur distalen Metaphyse sind Spontankorrekturen im Schaftbereich nur begrenzt möglich und verbleibende Achsabweichungen insbesondere bezüglich der Umwendbewegung häufig funktionell bedeutsam. Unabhängig von Alter und Frakturtyp wird deshalb eine Achsabweichung von $10^{\circ}$ in allen Ebenen als Grenze des konservativen Vorgehens festgelegt.

\section{Refrakturrisiko bei Grünholzfraktur}

Obwohl die Grünholzfraktur, bei der lediglich eine Kortikalis unterbrochen ist, per se eine stabile Frakturform darstellt, ist sie bei der Versorgung problembehaftet. Bei der belassenen und, z. B. weil wenig dislozierten, konservativ versorgten Grünholzfraktur kommt es in bis zu 30\% der Fälle zu einer Refraktur innerhalb 1 Jahrs [7]. Dies liegt am fehlenden Heilungsreiz im Bereich der nicht unterbrochenen Kortikalis.

Über dieses Refrakturrisiko muss aufgeklärt werden. Es stellt auch bei wenig dislozierten Frakturen eine relative Indikation zur operativen Versorgung dar. $\mathrm{Ob}$ man sich zu einer solchen auch bei geringer Dislokation entscheidet, muss gemeinsam mit den Eltern und dem $\mathrm{Pa}$ tienten in Ruhe besprochen werden. Gegen die Reposition und Osteosynthese (s. unten) sprechen das, wenn auch geringe, Narkoserisiko und die Gefahr von iatrogenen Verletzungen. Einen Eingriff befürwortend sind das ansonsten hohe Refrakturrisiko sowie die Möglichkeit der gipsfreien Behandlung nach Osteosynthese. 


\section{Elastisch-stabile intramedulläre Nagelung (ESIN)}

Bei allen Unterarmschaftfrakturen mit einer Dislokation von mehr als $10^{\circ}$ besteht die Indikation zur Reposition. Insbesondere bei isolierten Ulnafrakturen muss zudem auf die Möglichkeit einer Radiusköpfchenluxation, also einer Monteggia-Fraktur, geachtet werden. Diese stellt selbstverständlich unabhängig vom Dislokationsausmaß stets eine Operationsindikation dar.

Vollständige Frakturen werden in Narkose reponiert und das Ergebnis mit einem intramedullären Nagel gesichert. Hierbei muss nur der frakturierte Knochen versorgt werden, falls der dazugehörende paarige Knochen intakt ist [1].

Grünholzfrakturen müssen intraoperativ durch Brechen der intakten Kortikalis in eine vollständige Fraktur überführt werden, da sonst das hohe Refrakturrisiko weiter besteht. Da dadurch eine instabile Fraktur entsteht, empfehlen wir ebenfalls die Osteosynthese mittels ESIN.

Auch Bowing-Frakturen sollten aufgrund der hohen Rückstellkräfte bei erfolgter Reposition mit einem Nagel versorgt werden.

Somit ergibt sich bei Repositionsindikation auch nahezu immer die Indikation zur Osteosynthese. Der intramedulläre Nagel stellt dabei in jedem (Wachstums-)Alter die ideale Osteosynthese dar. Lediglich bei der extrem seltenen ( $2 \%$ im eigenen Patientengut) offenen Reposition kann eine Plattenosteosynthese erwogen werden, wenn bereits ein entsprechender Zugang gewählt wurde. Aus unserer Sicht stellt aber auch hier der intramedulläre Nagel die bessere Alternative dar, da das Osteosyntheseergebnis ebenso sicher, die Fraktur ebenso übungsstabil versorgt und das Weichteiltrauma und die NarbengröBe deutlich geringer sind.

\section{Problematik der diametaphysären Frakturen}

Frakturen am Übergang von der Dia- zur Metaphyse stellen für jeden Kindertraumatologen eine besondere Herausforderung dar. Der diametaphysäre Übergang ist in diesem Zusammenhang nicht genau definiert und ergibt sich eher aus der schwierigen operativen Versorgungsmöglichkeit.

Diese Frakturen haben ein geringes, mehr der Diaphyse zuzuordnendes Korrekturpotenzial. Bei ihrer Versorgung ist die Kirschner-Draht-Osteosynthese nicht mehr möglich, da sich die Gegenkortikalis nicht fassen lässt. Mit der ESIN wird wiederum nicht die notwendige 3-Punkt-Abstützung erreicht, was zu einer noch stärkeren Dislokation führen kann (• Abb.4).

Bei dieser problematischen Frakturregion herrscht bisher kein Konsens über das ideale Osteosyntheseverfahren. Von einigen Autoren - wie auch von uns wird die transepiphysäre intramedulläre Drahtosteosynthese ([5], $\boldsymbol{\square}$ Abb. 5) bevorzugt. Andere führen bei dieser Verletzung eine Plattenosteosynthese durch, die hier ausnahmsweise ihre Berechtigung hat $[9,11]$.

\section{Fazit für die Praxis}

Kindliche Frakturen des distalen Unterarmes haben ein enormes Korrekturpotenzial, das regelmäßig selbst vom erfahrenen Behandler unterschätzt wird. Es ist stets notwendig, sich dies vor Augen zu führen und auch bei den seltenen Indikationen zur Operation die Kirschner-Draht-Osteosynthese als wenig invasive Methode zu wählen.

- Bei Unterarmschaftfrakturen ist die Korrekturfähigkeit gering, ihre Behandlung ist die Domäne der ESIN.

- Die Grünholzfraktur ist bezüglich der Frakturform, der Operationsindikation und dem Refrakturrisiko als Besonderheit anzusehen.

- Einen Problembereich stellt der diametaphysäre Übergang dar. Hier sind die Frakturen häufig versorgungsbedürftig, die bisher genannten Osteosynthesemethoden jedoch oft insuffizient. Ausnahmsweise kann bei Frakturen in dieser Lokalisation eine Plattenosteosynthese durchgeführt werden.

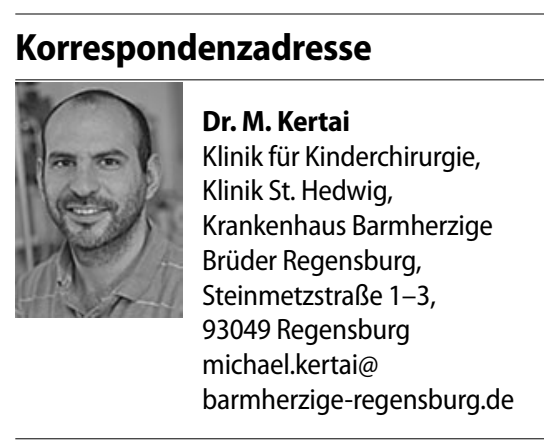

\section{Einhaltung ethischer Richtlinien}

Interessenkonflikt. M. Kertai und B. Reingruber geben an, dass kein Interessenkonflikt besteht.

Dieser Beitrag beinhaltet keine Studien an Menschen oderTieren.

The supplement containing this article is not sponsored by industry.

\section{Literatur}

1. Dietz H-G, Schmittenbecher PP, Slongo T et al (2006) Case collection of forearm fractures. In: Dietz H-G, Schmittenbecher PP, Slongo T, Wilkins KE (Hrsg) Elastic stable intramedulary nailing (ESIN). Thieme, Stuttgart New York, S 71-108

2. Kraus R, Schneidmüller D, Röder C (2005) Häufigkeit von Frakturen der langen Röhrenknochen im Wachstumsalter. Dtsch Arztebl 102(12):A838A842

3. Kraus R, Ploss C, Staub L et al (2006) Fractures of long bones in children and adolescents. Osteo Trauma Care 14:39-44

4. Kraus R, Reyers J, Alt V et al (2011) Physiological closure of the physeal plate of the distal radius: an MRI analysis. Clin Anat 24(8):1010-1015

5. Lieber J, Schmid E, Schmittenbecher PP (2010) Unstable diametaphyseal forearm fractures: transepiphyseal intramedullary Kirschner-wire fixation as a treatment option in children. Eur J Pediatr Surg 20(6):395-398

6. Laer L von, Kraus R, Linhart WE (2007) Korrekturmechanismen des wachsenden Skeletts. In: Laer L von, Kraus R, Linhart WE (Hrsg) Frakturen und Luxationen im Wachstumsalter. Thieme, Stuttgart New York, S 12-17

7. Laer L von, Kraus R, Linhart WE (2007) Frakturen im Bereich des Vorderarmschaftes. In: Laer L von, Kraus R, Linhart WE (Hrsg) Frakturen und Luxationen im Wachstumsalter. Thieme, Stuttgart New York, S 192-210

8. Ogden JA (2000) Radius and ulna. In: Ogden JA, Hensinger RN, McCollough N (Hrsg) Skeletal injury in the child. Springer, Berlin Heidelberg New York, S 567-605

9. Reis WL van der, Otsuka NY, Moroz P et al (1998) Intramedullary nailing versus plate fixation for unstable forearm fractures in children. J Pediatr Orthop 18:9-13

10. Shapiro F, Holtrop ME, Glimcher MJ (1977) Organization and cellular biology of the perichondrial ossification groove of Ranvier: a morphological study in rabbits. J Bone Joint Surg Am 59(6):703-723 


\section{Kindertraumatologie}

11. Vainionapp S, Bostman O, Rokkanen P (1987) Internal fixation of forearm fractures in children. Acta Orthop Scand 58:121-123

12. Weinberg $A-M$, Altermatt $S$, Hell $A$, Reilmann H (2006) Distaler Unterarm. In: Weinberg A-M, Tscherne H (Hrsg) Tscherne Unfallchirurgie: Unfallchirurgie im Kindesalter, Teil 1. Springer, Berlin Heidelberg New York, S 362-384

13. Weinberg A-M, Altermatt S, Hell A, Reilmann $\mathrm{H}$ (2006) Epiphysäre Frakturen des distalen Radius. In: Weinberg A-M, Tscherne H (Hrsg) Tscherne Unfallchirurgie: Unfallchirurgie im Kindesalter, Teil 1. Springer, Berlin Heidelberg New York, S 384-387 